\title{
A Simple Analytic Treatment of the Intergalactic Absorption Effect in Blazar Gamma-Ray Spectra
}

\author{
F.W. Stecker \\ NASA/Goddard Space Flight Center \\ stecker@milkyway.gsfc.nasa.gov \\ S.T. Scully \\ Department of Physics, James Madison University \\ scullyst@jmu.edu
}

Received —

Astrophysical Journal Letters 652, L9 (2006) 


\begin{abstract}
We derive a new and user friendly simple analytic approximation for determining the effect of intergalactic absorption on the $\gamma$-ray spectra of $\mathrm{TeV}$ blazars the energy range $0.2 \mathrm{TeV}<E_{\gamma}<2 \mathrm{TeV}$ and the redshift range $0.05<z<0.4$. In these ranges, the form of the absorption coefficient $\tau\left(E_{\gamma}\right)$ is approximately logarithmic. The effect of this energy dependence is to steepen intrinsic source spectra such that a source with an approximate power-law spectral index $\Gamma_{s}$ is converted to one with an observed spectral index $\Gamma_{o} \simeq \Gamma_{s}+\Delta \Gamma(z)$ where $\Delta \Gamma(z)$ is a linear function of $z$ in the redshift range $0.05-0.4$. We apply this approximation to the spectra of $7 \mathrm{TeV}$ blazars.
\end{abstract}

Subject headings: Gamma-rays: general - blazars: individual (Mkn 180, PKS 2005-489, PKS 2155-304, H 2356-309, 1ES 1218+30, 1ES 1101-232, PG $1553+113)$

\title{
1. Introduction
}

Stecker, Malkan \& Scully (2006) (SMS) have used recent Spitzer observations (Le Floc'h et al. 2005, Perez-Gonzalez et al. 2005) along with other data on galaxy luminosity functions and redshift evolution in order to make a detailed evaluation of the intergalactic photon density as a function of both energy and redshift for $0<z<6$ for photon energies from $.003 \mathrm{eV}$ to the Lyman limit cutoff at $13.6 \mathrm{eV}$ in a $\Lambda$ CDM universe with $\Omega_{\Lambda}=0.7$ and $\Omega_{m}=0.3$. They then used their calculated intergalactic photon densities to calculate the optical depth of the universe, $\tau$, for $\gamma$-rays having energies from $4 \mathrm{GeV}$ to $100 \mathrm{TeV}$ emitted by sources at redshifts from 0 to 5 . They also gave a parametric fit with numerical coefficients for $\tau\left(E_{\gamma}, z\right)$. 
As an example of the application of the detailed numerical determination of the optical depth, they calculated the absorbed spectrum of the blazar PKS 2155-304 at $z=0.117$ and compared it with the spectrum observed by the H.E.S.S. air Cherenkov $\gamma$-ray telescope array. It was noted that a steepening in an $E^{-2}$ power-law differential photon source spectrum for PKS 2155-304 would be steepened by approximately one power to $E^{-3}$ as would be produced by a $\gamma$-ray opacity with a logarithmic energy dependence.

The purpose of this letter is to generalize this result by determining fits for approximating $\tau\left(E_{\gamma}, z\right)$ by logarithmic functions in order to predict power-law steepenings in assumed blazar source spectra of the form $E^{-\Gamma_{s}}$ to observed spectra of the form $E^{-\Gamma_{o}}$ where $\Gamma_{o} \simeq \Gamma_{s}+\Delta \Gamma$ and $\Delta \Gamma$ is determined to be a linear function of $z$.

\section{Calculation}

In order to derive our results, we fit the results from SMS for $\tau\left(E_{\gamma}, z\right)$ to a form which is assumed to be logarithmic in $E_{\gamma}$ in the energy range $0.2 \mathrm{TeV}<\mathrm{E}_{\gamma}<2 \mathrm{TeV}$ and which has a linear dependence on $z$ over the range $0.05<z<0.4$. It is important to note that our linear fit to the $z$ dependence is both qualitatively and quantitatively different from the linear dependence on redshift which would be obtained for small redshifts $z<<1$ and which simply comes from the fact that for small $z$ where luminosity evolution is unimportant and where $\tau \propto d$, with the distance $d \simeq c z / H_{0} \propto z$, our quantitaitve fit for the higher redshift range $0.05<z<0.4$ comes from the more complex calculations based on the models of SMS. For this reason, our linear fits are not simply proportional to redshift.

We choose the form of our fitting function inn both redshift and energy to be given by

$$
\tau\left(E_{\gamma}, z\right)=(A+B z)+(C+D z) \ln \left[E_{\gamma} /(1 \mathrm{TeV})\right],
$$


where $A, B, C$ and $D$ are constants.

If we then postulate an intrinsic source spectrum which can be approximated by a power law over this limited energy range of one decade,

$$
\Phi_{s}\left(E_{\gamma}\right) \simeq K E_{\gamma}^{-\Gamma_{s}}
$$

the spectrum which will be observed at the Earth following intergalactic absorption will be of the power-law form

$$
\Phi_{o}\left(E_{\gamma}\right)=K e^{-(A+B z)} E_{\gamma}^{-\left(\Gamma_{s}+C+D z\right)}
$$

This can be compared with the empirically observed spectrum which is usually presented in the literature to be of the power-law form. The observed spectral index, $\Gamma_{o}$, will then be given by

$$
\Gamma_{o}=\Gamma_{s}+\Delta \Gamma(z)
$$

where the intrinsic spectral index of the source is steepened by $\Delta \Gamma(z)=C+D z$.

Table 1. Optical Depth Parameters

\begin{tabular}{ccccc}
\hline \hline Evolution Model & A & B & C & D \\
\hline Fast Evolution & -0.475 & 21.6 & -0.0972 & 10.6 \\
Baseline & -0.346 & 16.3 & -0.0675 & 7.99 \\
\hline
\end{tabular}


The parameters $A, B, C$, and $D$ obtained by fitting the optical depths derived for the fast evolution (FE) and baseline (B) models of SMS are given in Table 1. Figures 1 and 2 show the excellent fits of these parameters to a linear dependence in both $\ln E_{\gamma}$ and redshift.

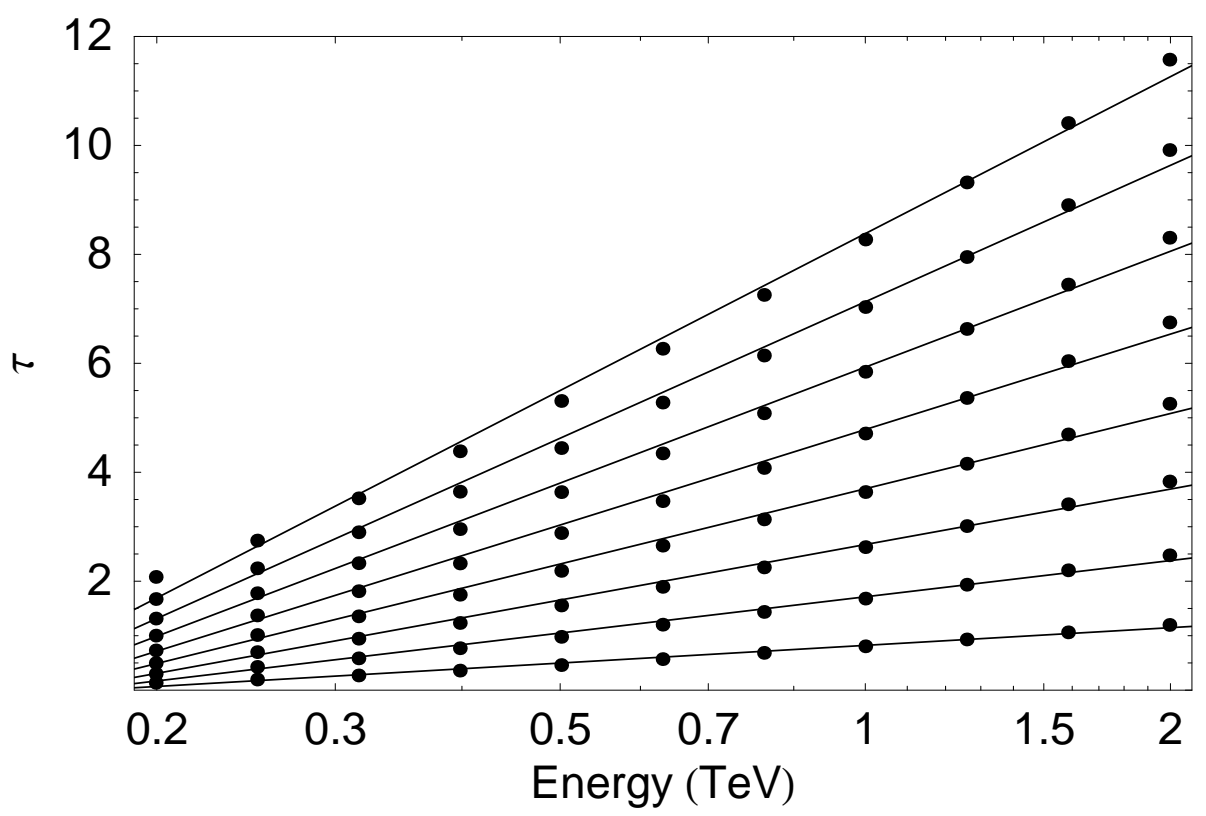

Fig. 1. - Linear fits in $\ln E_{\gamma}$ to the optical depths calculated in SMS (points shown) for their fast evolution (FE) case in the energy range $0.2 \mathrm{TeV}$ to $2.0 \mathrm{TeV}$ for redshifts of (bottom to top) $0.05,0.10,0.15,0.20,0.25,0.30,0.35$, and 0.40 . A similar result is obtained for the SMS baseline (B) case.

\section{Spectral Indices of Individual Sources}

Table 2 gives a list of blazars which have been detected at $\mathrm{TeV}$ energies and for which spectral indices $\left(\Gamma_{o}\right)$ have been measured in the energy range $0.2-2 \mathrm{TeV}$. We also give the observed redshifts of these sources and the intrinsic spectral indices of the sources $\left(\Gamma_{s}\right)$ derived from the baseline (B)and fast evolution (FE) models using our analytic expressions for $\Delta \Gamma(z)$. A spectral index of less than 2 indicates that the energy range of the observation 


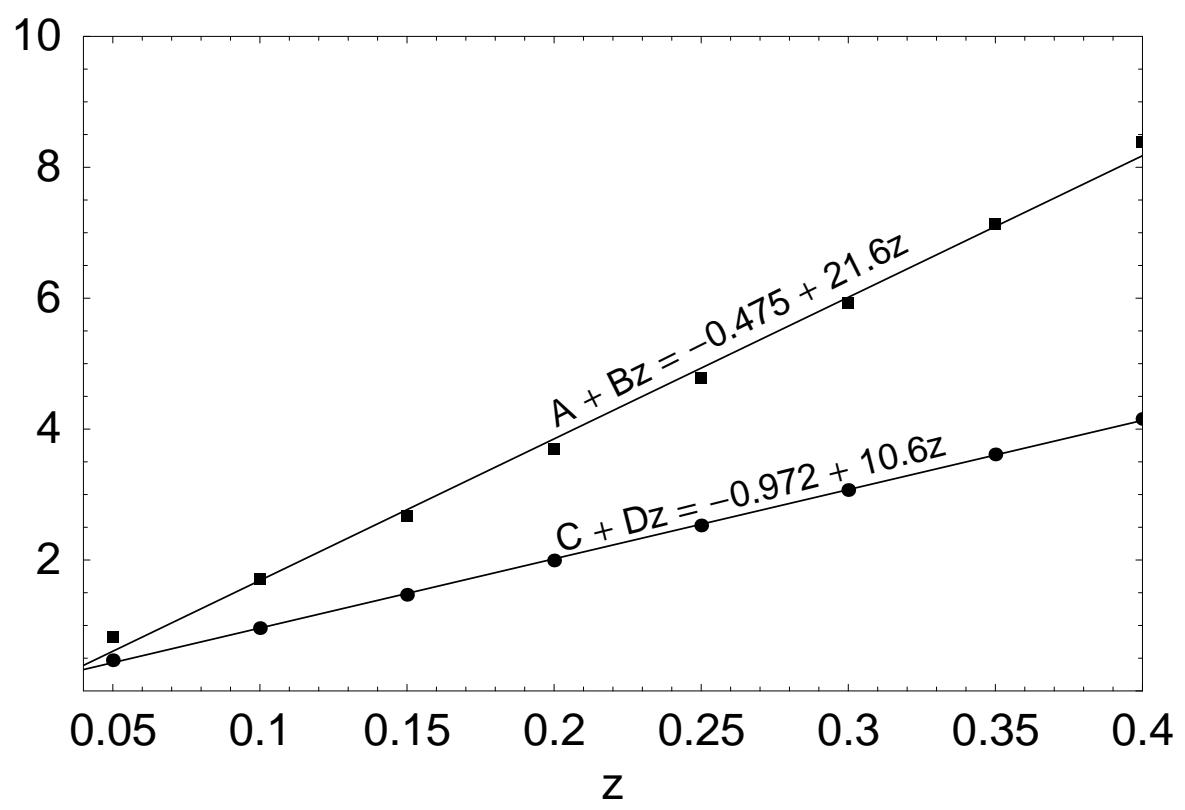

Fig. 2. - The fits obtained for the linear functions $A+B z$ and $C+D z$ shown for the FE case as descibed in the text. A similar result is obtained for the B case.

would be below the Compton peak energy in the spectral energy distribution, $E_{\gamma}^{2} \Phi\left(E_{\gamma}\right)$ of a synchrotron self-Compton (SSC) model (Stecker, De Jager \& Salamon 1996). In the case of the blazar PG $1553+113$, the energy range of the observation is from 0.09 to $0.4 \mathrm{TeV}$ which is somewhat below the energy range we have used in deriving our approximation.

\section{Conclusions}

We have derived a new and user friendly simple analytic approximation for determining the effect of intergalactic absorption on the spectra of $\gamma$-ray sources in the energy range 0.2 $\mathrm{TeV}<E_{\gamma}<2 \mathrm{TeV}$ and the redshift range $0.05<z<0.4$, where the absorption is primarily from interactions with intergalactic photons in the optical to near infrared wavelength range. In these energy and redshift ranges, the form of the absorption coefficient $\tau\left(E_{\gamma}\right)$ is approximately logarithmic. The effect of this energy dependence is to steepen the intrinsic 
Table 2. Spectral Indices for Seven Observed Blazars in the $0.2-2$ TeV Energy Range

\begin{tabular}{ccccc}
\hline \hline Source & $z$ & $\Gamma_{o}{ }^{*}$ & $\Gamma_{s}(\mathrm{~B})$ & $\Gamma_{s}(\mathrm{FE})$ \\
\hline Mkn 180 & 0.045 & $3.3(\mathrm{a})$ & 3.0 & 2.9 \\
PKS 2005-489 & 0.071 & $4.0(\mathrm{~b})$ & 3.5 & 3.4 \\
PKS 2155-304 & 0.117 & $3.3(\mathrm{c})$ & 2.4 & 2.2 \\
H 2356-309 & 0.165 & $3.1(\mathrm{~d})$ & 1.9 & 1.5 \\
1ES 1218+30 & 0.182 & $3.0(\mathrm{e})$ & 1.6 & 1.2 \\
1ES 1101-232 & 0.186 & $2.9(\mathrm{f})$ & 1.5 & 1.0 \\
PG 1553+113 & $0.36^{\dagger}$ & $4.2(\mathrm{~g})$ & 1.4 & 0.5 \\
\hline
\end{tabular}

*MAGIC and H.E.S.S.references for observed spectral indices denoted by letters are (a) Albert et al. (2006b), (b) Aharonian et al. (2005b), (c) Aharonian et al. (2005a), (d) Aharonian et al. (2006a), (e) Albert et al. (2006a), (f) Aharonian et al. (2006b), (g) Albert et al. (2006b).

${ }^{\dagger}$ Although Albert et al. (2006b) and Aharonian et al. (2006c) use assumptions about the source spectrum of this object to place upper limits on $z$ of 0.78 and 0.74 respectively, the catalogued redshift of $\mathrm{PG} 1553+113$ is 0.36 (Hewitt and Burbidge 1989). 
source spectrum such that a source with an approximate power-law spectral index $\Gamma_{s}$ is converted to one with an observed spectral index $\Gamma_{o} \simeq \Gamma_{s}+\Delta \Gamma$ in the energy range $0.2-2 \mathrm{TeV}$, where $\Delta \Gamma(z)$ is a linear function of $z$ in the redshift range $0.05-0.4$. We have applied this approximation to the spectra of seven TeV blazars. These power-law approximations, both observational and theoretical, are only useful over the limited energy range indicated, viz. $0.2-2 \mathrm{TeV}$. The actual spectra should exhibit some curvature. At higher energies the spectra should cut off either because of a natural upper limit to the source spectra or, more likely in the case of high frequency peaked BL Lac objects (HBLs), because of the increase in optical depth with energy owing to interactions with the much more numerous intergalactic far infrared photons. A full numerical treatment of absorption can be made using the exact form of the optical depth as a function of redshift and energy as calculated in the detailed work of SMS. Examples of more detailed treatments of effects of intergalactic $\gamma$-ray absorption are given for Mkn 501 and Mkn 421 by Konopelko et al. (2003) and for PKS 2155-304 by SMS. However, because of the limited energy range of empirical $\mathrm{TeV}$ spectral determinations, and because of the significant statistical and systematic uncertainties in the empirical determinations of spectral indices of TeV sources, our approximate linear relations can be quite useful in making rapid and simple analyses of TeV source spectra. 


\section{REFERENCES}

Aharonian, F. et al. 2005a, A \& A 430, 865

Aharonian, F. et al. 2005b, A \& A 436, L17

Aharonian, F. et al. 2006a, e-print astro-ph/ 0607569

Aharonian, F. et al. 2006b, Nature 440, 1018

Aharonian, F. et al. 2006c, A \& A 448, L19

Albert, J. et al. 2006a, ApJ 642, L119

Albert, J. et al. 2006b, e-print astro-ph/ 0606161

Albert, J. et al. 2006c, e-print astro-ph/ 0606630

Hewitt, A. \& Burbidge, G. 1989, A New Optical Catalogue of Quasi-Stellar Objects (Chicago: Univ. Chicago Press).

Le Floc'h, E.et al. 2005, ApJ 632, 169

Pérez-González, P.G. et al. 2005, ApJ 630, 82

Stecker, F. W., De Jager, O. C. \& Salamon, M. A. 1996, ApJ 473, L75

Stecker, F. W., Malkan, M. A. \& Scully, S. T. 2006, ApJ 648, in press, e-print astro-ph/ 0510449 